\title{
KẾT HợP CÁC PHÉP TOÁN HÌNH THÁl HỌC VÀ LÀM MÁNH ĐẾ NÂNG CAO CHẤT LƯợNG ÁNH ĐƯỜNG NÉT
}

\author{
ĐỖ NĂNG TOÀN, NGÔ QUỐC TẠO *
}

\begin{abstract}
This paper present a method of using morphology operations such as Dilation. Erosion and thinning technique for improving feature images. In general, the morphology operations are used for increasing or reducing the thickness of lines, smoothing contours or filling holes in images. Unfortunately, the erosion operation creates dot lines. In order to overcome this shortcoming, we use the dilation operation and thinning technique step by step. This approach is useful for reducing the thickness of lines and keeps the connectness of lines. The first points of LOOP are suitable chosen for process speed. The paper also shows kinds of maps that can be vectorized and have been tested by the software package MAPSCAN developed in the Department of Pattern Recognition and Knowledge Engineering such as:

- Topography, hydrography and transport maps etc...

- Technical, designing and electronic circle drawings, finger images etc...
\end{abstract}

\section{GIỚT THIڤ̣̂U}

Trong xử lý và nhận dạng ånh, có một số loại ảnh đường nét gồm các đối tượng (objects) là các đường cong có độ dài lón hơn nhiều so với đồ dày của nó, ví dụ như là ánh các ký tự, dấu vân tay, sơ đồ mạch địện tử, bản vẽ kỹ thuật, bản đồ v.v.. Để xử lý các loại ảnh này người ta thường xây dựng các hệ mô phơng theo cách phân tích ảnh của con người gọi là hệ thống thị giác máy (Computer Vision System). Có nhiều hệ thống được cài đặt theo phương pháp này (xem hình 1) như hệ nhận dạng chữ viết băng thiết bị quang học OCR (Optical Character Recognition), hệ thống nhận dạng vân tay AFIS (Automated Fingerprint Identification System) v.v..

Có nhiều phương pháp trích chọn đặc điểm được biết tới như phương pháp sử dụng bộ lọc sóng ngăn (Wavelet), sử dụng hệ số Fourier, sử dụng các mô men bất biến, sử dụng các đặc trưng cưa biên như tính trơn và các điểm đặc biệt, sử dụng các đặc trưng tô pô dựa trên xương của đường nét... Phương pháp trích chọn đặc điểm sử dụng ảnh đã mánh được sử dựng nhiều vì việc trích chọn đặc điểm trở nên dễ dàng. Sau bước này các đường nét đã mảnh được véc tơ hóa ánh phục vụ việc nén dữ liệu, nhằm làm giảm thiểu yêu cầu về không gian lưu trữ, xử lý và thời gian xử lý. Kỹ thuật "làm mảnh" là một trong nhiều úng dụng của phép toán hình thái học (morphology).

Thông thường các thuật toán làm mảnh thường bao gồm nhiều lần lặp, trong mỗi lần lặp tất cá các điểm của đối tượng sẽ được kiểm tra nếu như chúng thóa mãn điều kiện

* Chương trình nhập bản đồ tự động đã được tài trợ và phát triển trong khuôn khổ của dự án UNFPA-INT 92/P23 "Phần mềm máy tính và trọ" giúp cho hoọt đậng dân sô". 
xóa nào đó tùy thuộc vào thuật toán thì nó sẽ bị xóa đi. Quá trình được lặp lại cho đến khi không còn điểm biên nào được xóa. Đối tượng được bóc dần lớp biên cho đến khi bị thu mánh lại thành một đường duy nhất có độ dày bằng 1 pixel. Nhưng trên thực tế, chẳng hạn khi sư dụng các phép toán hình thái nhăm lấp đầy các lỗ hỗng, làm trơn biên và nối số đường đứt nét, đôi khi ta chỉ cần bóc một số lớp nhất định để làm mánh đối tượng đến một độ nhất định và bán thân trong mỗi phần trong cùng một ánh lại cần làm mánh với một số lóp khác nhau.

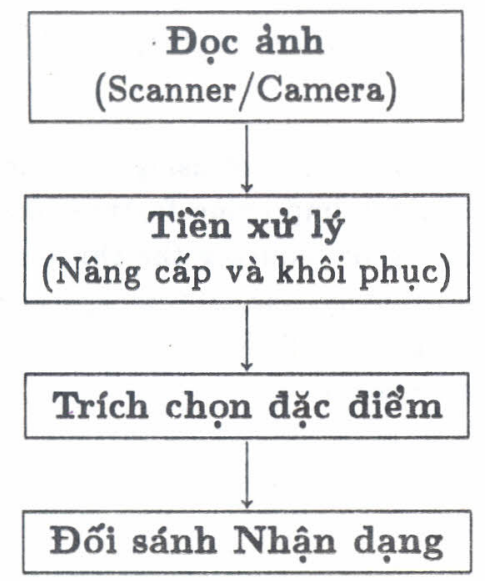

Hinh 1. Mô hình tổng quát của hệ thống nhận dạng ánh

Bài này đưa ra phương pháp chọ các điểm LOOP trong quá trình làm mánh một cách tự động dựa theo các chu trình LOOP được sư dụng trong thuật toán "Làm mánh" [5], giúp cho việc làm mánh đối tượng với số lớp nhất định không bị ánh hướng bởi số lần thực hiện làm mánh.

Phần còn lại của bài báo như sau: Phần 2 giới thiệu các phép toán hình thái học. Phần 3 , Phần 4 là các kết quá thực nghiệm. Phần cuối cùng là kết luận.

\section{CÁC PHÉP TOÁN HİNH THÁI}

\subsection{Một số định nghĩa}

"Hình thái" là thuật ngữ chỉ sự nghiên cứu về cấu trúc hay hình học tô pô của đối tượng trong ảnh. Biến đổi "hình thái" có rất nhiều ứng dụng, mà một trong nhũng ứng dụng quan trọng là "làm mánh" (Thining).

Phần lớn các phép toán "hình thái” được định nghĩa từ hai phép toán cơ bản là phép "dãn nơ" (Dilation) và phép "co" (Erosion). Các phép toán này được định nghĩa như sau:

Giả thiết ta có đối tượng $X$ và phần tữ cấu trúc $B$ trong không gian Euclide hai chiều. Kí hiệu $B_{x}$ là dịch chuyển của $B$ tới vị trí $x$.

Định nghĩa 1 (Dilation [1]). Phép "dãn nơ” của $X$ theo cấu trúc $B$ là tập hợp của tất cá các điềm $x$ sao cho $B_{x}$ chạm tới $X$

$$
X \oplus B:=\left\{x: B_{x} \cap X \neq \emptyset\right\} .
$$

Định nghĩa 2 (Erosion [1]). Phép "co" của $X$ theo $B$ là tập hợp tất cá các điễm $x$ sao cho $B_{x}$ nằm trong $X$ 


$$
X \ominus B:=\left\{x: B_{x} \subset X\right\} .
$$

\subsection{Một số tính chất cùa phép biển đồi hình thái}

- Tính chất bất biến

$$
\begin{aligned}
& ((X \oplus B) \ominus B) \oplus B=X \oplus B \\
& ((X \ominus B) \oplus B) \ominus B=X \ominus B
\end{aligned}
$$

- Tính chất phân bố của phép toán hình thái đối với tập cấu trúc

$$
\begin{aligned}
& X \oplus\left(B \cup B^{\prime}\right)=(X \oplus B) \cup\left(X \oplus B^{\prime}\right) \\
& X \ominus\left(B \cup B^{\prime}\right)=(X \ominus B) \cap\left(X \ominus B^{\prime}\right)
\end{aligned}
$$

- Tính chất phân bố của co đối với phép giao hai tập hợp

$$
(X \cap Z) \ominus B=(X \ominus B) \cap(Z \ominus B)
$$

- Tính kết hợp của phép toán co, dãn nớ

$$
\begin{aligned}
& (X \oplus B) \ominus B^{\prime}=X \ominus\left(B \oplus B^{\prime}\right) \\
& (X \oplus B) \oplus B^{\prime}=X \oplus\left(B \oplus B^{\prime}\right)
\end{aligned}
$$

- Tính chất gia tăng

$$
\begin{aligned}
X \subset X^{\prime} \Rightarrow & X \ominus B \subset X^{\prime} \ominus B \quad \forall B \\
& X \oplus B \subset X^{\prime} \oplus B \quad \forall B \\
B \subset B^{\prime} \Rightarrow & X \ominus B \subset X \ominus B^{\prime} \quad \forall X
\end{aligned}
$$

- Tính chất đối ngẫu

$$
X \oplus B=(X \ominus B)^{c}
$$

\subsection{Làm mảnh dưới góc độ xử lý "hình thái”}

Chúng ta có thể dùng các phép toán co, dãn để làm mảnh các ảnh.

Định nghĩa 3. Trong xử lý "hình thái", phép toán "làm mảnh" đự̛̣c định nghĩa như sau:

$$
X \circ B:=X \backslash(X \otimes B)
$$

Trong đó: $B$ là phần tứ cấu trúc dùng trong làm mảnh, $\otimes$ là toán tử trúng - trượt (hit - miss operator).

Ơ đây "trúng" có thể hiểu khi đó phần chung giao nhau của $B$ với $X$ là không rỗng.

$$
X \otimes B:=\left(X \ominus B_{\mathrm{ob}}\right) \backslash\left(X \oplus B_{\mathrm{bk}}\right)
$$

với: $B_{\mathrm{ob}}$ - tập các điểm của $B$ thuộc vào đối tượng,

$B_{\mathrm{bk}}$ - tập các điểm của $B$ thuộc biên. 
Đễ có được kết quá chính xác, việc làm mánh cần phải được thực hiện một cách đối xứng. Do đó, người ta thường định nghĩa dãy các phần tử cấu trúc

$$
\{B\}:=\left\{B^{i}, 1<i<n\right\},
$$

trong đó $B^{i}$ là $B^{i-1}$ được quay đi một góc và được sứ dụng lần lượt theo trình tự

$$
X \bigcirc\{B\}:=\left(\left(\ldots\left(\left(X \circ B^{1}\right) \circ B^{2}\right) \ldots\right) \circ B^{n}\right)
$$

Định nghĩa 4. Tập $X$ được gọi là mánh đối với cấu trúc $B$ nếu $X O\{B\}=X$.

\section{Thuật toán làm månh:}

Bước 1: Vào ma trận ành $X$.

Bước 2: $X \leftarrow X O\{B\}$.

Bước 3: Nếu $X=X \mathrm{O}\{B\}$ thì dừng; nếu khác đi thì quay lại bước 2 .

Mệnh đề 2.1. Thuật toán làm mánh dừng và cho kết quả là mảnh đối với cấu trúc $B$.

Chưing minh. Chúng ta có $X O\{B\} \subseteq X$ nên sau mỗi bước làm mánh số điểm trong $X$ giám đi. Do số phần tư $X$ khác không nên số lần thực hiện bước 2 không vượt quá số điểm cưa $X$. Do đó thuật toán làm mánh dừng.

Ví du : Đối với thuật toán Toumazet [8] thì tập $B$ được chọn như sau

$$
B=\begin{array}{lll}
0 & & 1 \\
0 & 1 & 1 \\
0 & & 1
\end{array}
$$

Nếu quay $B$ đi một góc $45^{\circ}$ chúng ta được các cấu trúc mới như sau:

$\begin{array}{llllllllllll}0 & 0 & & 0 & 0 & 0 & & 0 & 0 & 1 & \\ 0 & 1 & 1 & & 1 & & 1 & 1 & 0 & 1 & 1 & 0 \\ & 1 & 1 & 1 & 1 & 1 & 1 & 1 & & 1 & & 0 \\ 1 & 1 & & 1 & 1 & 1 & & 1 & 1 & 0 & & 1 \\ 1 & 1 & 0 & & 1 & & 0 & 1 & 1 & 0 & 1 & 1 \\ & 0 & 0 & 0 & 0 & 0 & 0 & 0 & & 0 & & 1\end{array}$

Như vậy chúng ta cần áp dụng làm mánh theo tám hướng sau: Đông, Đông Nam, Nam, Tây Nam, Tây, Tây Bắc, Bắc và Đông Bắc.

\section{LÀM MÁNH THEO CÁC ĐIỂM CHU TUYẾN}

\subsection{Phương pháp luận làm mành}

Đã có nhiều thuật toán làm mánh như trong các tài liệu [2-9]. Các thuật toán này làm mánh đều dựa trên tư tưởng chư yếu là xóa các điểm biên thỏa mãn điều kiện được xác định trước $[2-5,8,9]$. Như vậy cho trước ánh $I$, ký hiệu $\mathrm{C}(I)$ là tập các điểm biên (countour) của $I$ còn $I n(I)$ là tập các điểm trong của $I$. Quá trình làm mânh thông thường duyệt tất cá các điểm xem có thỏa mãn điều kiện xóa không. Nếu điểm nào thóa mãn điều kiện xóa thì chúng ta xóa ngay điểm này. Như vậy mối lần chúng ta phải duyệt toàn bộ các điểm ảnh. Xong việc xóa các điểm chỉ xáy ra trên biên ánh. Do đó số điểm cần xét trong mỗi lần xóa tối thiểu bằng số điểm của $\mathrm{C}(I)$. Do đó chúng ta có mệnh đề sau: 
Mệnh đề 3.1. Số điểm ít nhất trong mỗi lần duyệt trong làm mánh ít nhất băng số điểm biền $C(I)$ cúa ánh $I$.

Trong phần này chúng tôi đề cập đến thuật toán làm mánh đã được đề xuất trong [5] và đưa ra cách sử dụng đầu vòng LOOP một cách phù hợp trong việc sư dụng thuật toán làm giảm độ dày của đường do phép dãn nở nhằm nâng cao chất lượng cửa biên ánh.

\subsection{Làm månh dựa trên biên}

Theo mệnh đề 3.1 thì thuật toán làm mảnh hũu hiệu là thực hiện làm mánh trên các điểm trên biên. Có nhiều thuật toán sừ dụng đường biên để làm mánh. Một trong thuật toán làm mảnh đã được các tác giả Ngô Quốc Tạo và Đặng Ngọc Đức đưa ra trong [5] chủ yếu dựa vào đường biên để làm mánh đối tượng.

Đầu tiên thuật toán sẽ dò xét tất cả chu trình (LOOP) có trong ảnh, lưu trữ vị trí các điểm đầu vào trong một máng gọi là mảng FIRST. Sau đó thuật toán sẽ bao gồm nhiều lần lặp. Mỗi lần lặp dựa vào các điểm đầu này nó sẽ dùng thuật toán dò biên để xét duyệt tất cả các điểm biên trong ảnh. Với mỗi điểm biên, nếu như nó thoá mãn điều kiện xóa thì nó sẽ bị xóa ngay lập tức, sau đó nó mới xét tiếp điểm biên mới. Sau mỗi lần lặp thuật toán sẽ tìm lại vị trí các điểm đầu của chu trình và lưu lại trong máng FIRST. Nếu như đi hết một chu trình mà không có điểm nào bị xóa thì chu trình đó sẽ được đánh dấu là "đã mảnh". Đến lần lặp sau chu trình nào được đánh dấu là "đã mánh" thì sẽ không bị xét nữa. Thuật toán sẽ lặp lại đến khi không còn điểm biên nào bị xóa sau mỗi lần lặp.

Trong [5] đã chứng minh thuật toán tuần tự mới đám bảo tính liên thông của ánh.

\subsection{Cài thiện chất lượng cưa biên trong làm mành một bước}

Trong [5] đã đưa ra phương pháp tìm lại vị trí của điểm đầu sau mỗi lần lặp nhưng không xóa vị trí đầu hiện tại. Chúng tôi cải tiến thuật toán này bằng cách sau mỗi lần lặp kiểm tra và nếu thóa mãn điều kiện xóa thì xóa đầu mút hiện hành và tìm lại vị trí đầu của các vòng lặp.

\section{THƯC NGHIÊM}

Chúng tôi đã cài đặt các phép toán hình thái học này trong hệ mềm MAPSCAN. Các phép toán này tó ra hữu hiệu đối với các bản đồ có các bản đồ địa hình, thưy văn, lãnh thô, dường giao thông,... Các hình $2,3,4$ cho kết quả thực hiện các phép toán co, dãn và làm mảnh.

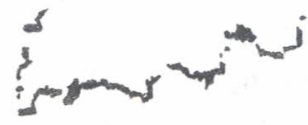

a) Trước khi dãn nơ.

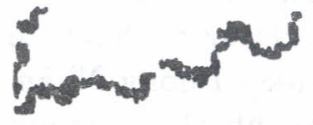

b) Sau khi dãn nở một bước

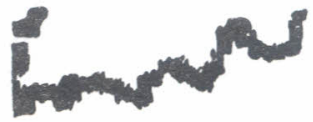

c) Sau khi dãn nở hai bước

Hinh 2. Kết quả cưa phép toán dãn nở 


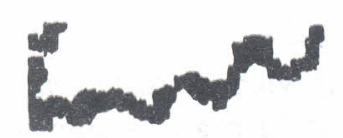

a) Trước khi co ảnh

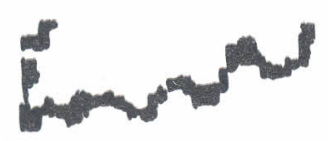

b) Sau khi co ảnh một bước

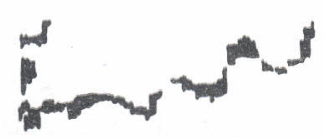

c) Sau khi co ảnh hai bước

Hình 9 . Kết quả cưa phép toán co

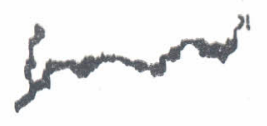

a) Trước khi làm mánh

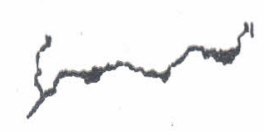

b) Làm mảnh một bước

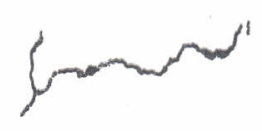

c) Làm mảnh hai bước

Hình 4. Kết quả cưa phép toán làm mảnh một số bước

Kểt quá hình 2 sau hai lần làm béo thì một đường đứt nét trở thành liền. Hình 3 chỉ ra sau 2 lần co ảnh thì một đường liền nét trờ nên đứt nét làm mất tính liên thông. Khăc phục nhược điểm đó, hình 4 cho thấy sau hai bước làm mảnh thì đường biên trở nên mánh và bảo đảm tính liên thông.

\section{KẾT LUẬA}

Trong bài này chúng tôi đầu tiên đề cập đển phương pháp sử dụng các phép toán hình thái để cải thiện ánh. Phép toán dãn nơ ảnh cho phép lấp đầy các lỗ hổng, làm trơn biên và nối một số đường đứt nét. Sau giai đọạn nối các đường đứt nét cần giảm độ dày cưa đường do phép toán dãn nở. Trong một số trường hợp thì nhược điểm của phép co ành là làm đứt nét các đường. Do đó chúng tôi cũng đề cập đến phương pháp làm mảnh có sử dụng các mặt nạ áp dụng các phép toán hình thái trên một số tập cấu trúc. Bài báo còn chî ra cách làm mánh tốt nhất là dùng các đường chu tuyến. Việc kết hợp khéo léo các phép toán hình thái sẽ làm cho các đường nét có chất lượng cao (trơn và ít lỗ hổng bên trong).

\section{Lời cám ơn}

Chúng tôi xin chân thành cám ơn GS TS Bạch Hưng Khang, PTS Lương Chi Mai đã đóng góp những ý kiến quí báu giúp chúng tôi hoàn thành công trình này. Chúng tôi cũng xin chân thành cảm ơn các đồng nghiệp Phòng Nhận dạng và Công nghệ tri thức đã tạo điều kiện thuận lợi cho chúng tôi nhanh chóng trong việc nghiên cứu cũng như việc cài đặt.

\section{TÀI LIỆU THAM KHẢO}

[1] Anil K. J., Fundamentals of Digital Image Processing, 1989, p. 384-388.

[2] Bạch Hưng Khang, Lương Chi Mai, Ngô Quốc Tạo, Đỗ Năng Toàn, et al., An Examination of 
Techniques for Raster-to-Vector Process and Implementation of Software Package for Automatic Map Data Entry-MapScan, Journal of Computer Science and Cybernetics 12 (2) (1996) 21-29.

[3] Bạch Hưng Khang, Lương Chi Mai, Ngô Quốc Tạo, Đỗ Năng Toàn, et al., An Examination of Techniques for Raster-to-Vector Process and Its Implementation-MapScan Package Software, International Symposium, AMPST96, University of Bradford, UK, 26-27 March, 1996.

[4] Bạch Hưng Khang, Lương Chi Mai, Ngô Quốc Tạo, Đỗ Năng Toàn, et al., MapScan for Windows - Software Package for Automatic Map Data Telecommunication Technologies (APSITT "97), Hanoi, Vietnam, March 13-14, 1997.

[5] Ngô Quốc Tạo, Đặng Ngọc Đức, Thuật toán làm mảnh tuần tự mới, Tuyển tập báo cáo Hội nghị khoa học Viện Công nghệ thông tin, Hà Nội, ngày 5-6 tháng 12, 1996.

[6] Đỗ Năng Toàn, Một kỹ thuật tương tác trong quá trình véc tơ hóa bản đồ, Tuyển tập báo cáo Hội nghị khoa học Viện Công nghệ thông tin, Hà Nội, ngày 5-6 tháng 12, 1996.

[7] Đỗ Năng Toàn, Một phương pháp giữ các điểm khớp trong véc tơ hóa bán tự động không qua làm mánh, Tạp chí Tin học và Diều khiển học 13 (4) (1997) 25-32.

[8] Toumazet J.J., Traitement de I'Image par Exemple, Symbex, Chaptre 5, Images Binaires Operateurs Morphologiques, pp. 117-139, 1990.

[9] Wang P.S.P. and Zhang Y.Y., A Fast and Flexible Thinning Algorithms, IEEE Transactions on Computer 38 (1989) 741-745.

Received: July 9, 1997

Viện Công nghệ thông tin, Trung tâm KHTN và CNQG. 